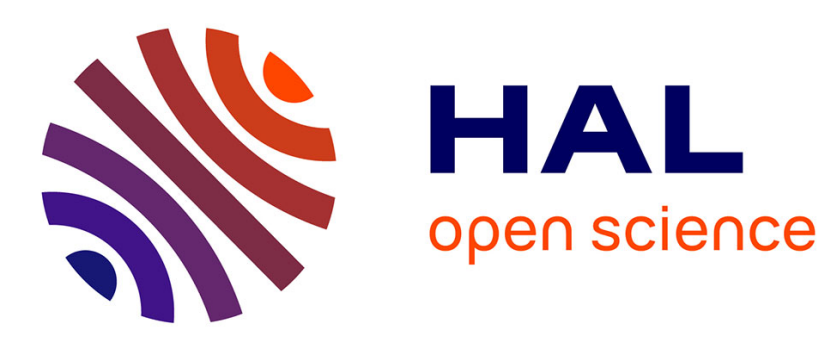

\title{
Study of the premixed flame model with heat losses. The existence of two solutions
}

\author{
Lionel Roques
}

\section{To cite this version:}

Lionel Roques. Study of the premixed flame model with heat losses. The existence of two solutions. European Journal of Applied Mathematics, 2005, 16 (6), pp.741-765. 10.1017/S0956792505006431 . hal-02681201

\section{HAL Id: hal-02681201 https://hal.inrae.fr/hal-02681201}

Submitted on 31 May 2020

HAL is a multi-disciplinary open access archive for the deposit and dissemination of scientific research documents, whether they are published or not. The documents may come from teaching and research institutions in France or abroad, or from public or private research centers.
L'archive ouverte pluridisciplinaire HAL, est destinée au dépôt et à la diffusion de documents scientifiques de niveau recherche, publiés ou non, émanant des établissements d'enseignement et de recherche français ou étrangers, des laboratoires publics ou privés. 
Version définitive du manuscrit publié dans / Final version of the manuscript published in :

European Journal of Applied Mathematics, 2005,16 (6), 741-765

\title{
Study of the premixed flame model with heat losses The existence of two solutions
}

\author{
Lionel Roques
}

October 16, 2007

\begin{abstract}
We study the existence of planar flames, in the case of a single-step chemical reaction with volumetric heat losses, with a general reaction term. We prove that for all positive Lewis numbers, and for small values of the heat loss rate parameter, two distinct solutions exist. We also give upper bounds for the flame speed and for the heat loss rate parameter. Moreover, we explicitly compute a lower bound for the unburned gases after reaction.
\end{abstract}

\section{Introduction and main results}

Zeldovitch showed in [16], using asymptotic methods, that the reaction-diffusion system modelling the propagation of a premixed laminar flame with heat losses has got two travelling-wave solutions in the case of high activation energies. More recently, Glangetas \& Roquejoffre in [7] demonstrated the same result, as a consequence of the dispersion relation which was obtained by Joulin \& Clavin $([9,10])$, and proved rigorously in $[7]$. The case of a more general nonlinearity has been studied by Giovangigli in [6], in which the author proves the existence of a solution for a fixed flame speed $c$ (considering that the minimum heat loss rate parameter $\lambda$ is an unknown of the problem), with a Lewis Number Le equal to 1. In this paper, we follow the framework of Berestycki, Nicolaenko \& Scheurer [3], who studied the adiabatic case $(\lambda=0)$; keeping $c$ as a solution of the problem, we prove the existence of two solutions for small values of $\lambda$. Also we establish that, in some cases, the solution with the greatest flame speed converges to the solution of the adiabatic problem as $\lambda \rightarrow 0$, whereas the other flame speed converges to 0 .

Furthermore, we compute some new bounds for the solutions. In particular, Giovangigli has proved in [6] that, when $L e=1$, the flame speed $c$ was bounded from above by the flame speed $c_{a d}$ of the adiabatic problem. Here we extend his result to $L e \leq 1$ (which is physically meaningful since, e.g., $L e=0.4$ for hydrogen), by showing that $c$ is smaller than the flame speed of an adiabatic 
Version définitive du manuscrit publié dans / Final version of the manuscript published in :

European Journal of Applied Mathematics, 2005, 16 (6), 741-765

scalar problem. Moreover, for all $L e>0$, we give an explicit upper bound for $c$. We also give a lower bound for the unburned gases after reaction.

Let $\Lambda$ and $\lambda$ be two positive real numbers. The aim of this work is to prove existence and nonexistence results for the following problem:

Find two nonnegative classical functions $u$ and $v$ and a nonnegative real number $c$ which satisfy

$$
\left\{\begin{aligned}
-u^{\prime \prime}+c u^{\prime} & =f(u, v)-\lambda h(u) \\
-\Lambda v^{\prime \prime}+c v^{\prime} & =-f(u, v)
\end{aligned} \quad \text { on } \mathbb{R},\right.
$$

with the boundary conditions

$$
\begin{cases}u(-\infty)=0, & u(+\infty)=0 \\ v(-\infty)=1, & v^{\prime}(+\infty)=0\end{cases}
$$

The following assumptions on $f$ will be made in the sequel: there exist two functions $p$ and $g$ such that

$$
f(u, v)=p(u) g(v) \text { in } \mathbb{R} \times \mathbb{R},
$$

where the function $p$ is globally Lipschitz continuous on $\mathbb{R}$, nondecreasing and of "ignition" type:

$$
\exists \theta \in(0,1) \text { s.t. } p(x)=0 \text { for all } x \leq \theta \text { and } p(x)>0 \text { for all } x>\theta,
$$

and the function $g$ is in $C^{0}(\mathbb{R})$, increasing on $\mathbb{R}_{+}$and such that

$$
g<0 \text { on }(-\infty, 0) \text { and } g(0)=0 \text {; }
$$

furthermore, for all $\gamma>0$, let us set

$$
k^{*}(\gamma):=\max \left\{\sup _{s \in(0,1)} \frac{g(\gamma s)}{g(s)}, \gamma\right\} \text { and } k_{*}(\gamma):=\min \left\{\inf _{s \in(0,1)} \frac{g(\gamma s)}{g(s)}, \gamma\right\},
$$

we then assume that

$$
\text { for all } \gamma>0,0<k_{*}(\gamma) \leq \gamma \leq k^{*}(\gamma)<+\infty \text {. }
$$

Hypothesis (6) is for instance satisfied by functions $g$ of the type $g(y)=y^{n}$, with $n>0$. It also works with functions $g$ such that there exists $n \geq 1, n \in \mathbb{N}$ such that $g \in C^{n}(\mathbb{R}) g^{(n)}(0) \neq 0$, where $g^{(n)}$ is the $n^{\text {th }}$ derivative of $g$.

The function $h$ is supposed to be in $C^{1}(\mathbb{R})$ and strictly increasing. Moreover, it satisfies

$$
h(0)=0, h(1)=1, \exists \alpha, \beta \in \mathbb{R} \text { s.t. } 0<\alpha \leq h^{\prime} \leq \beta .
$$

We call $\left(u_{a d}, v_{a d}, c_{a d}\right)$ the solution of the following problem without heat loss (see [3] for the existence of such solutions):

$$
\left\{\begin{aligned}
-u_{a d}^{\prime \prime}+c_{a d} u_{a d}^{\prime} & =f\left(u_{a d}, v_{a d}\right) \\
-\Lambda v_{a d}^{\prime \prime}+c_{a d} v_{a d}^{\prime} & =-f\left(u_{a d}, v_{a d}\right)
\end{aligned} \quad \text { on } \mathbb{R},\right.
$$


Version définitive du manuscrit publié dans / Final version of the manuscript published in :

European Journal of Applied Mathematics, 2005, 16 (6), 741-765

with the boundary conditions

$$
\begin{cases}u_{a d}(-\infty)=0, & u_{a d}(+\infty)=1, \\ v_{a d}(-\infty)=1, & v_{a d}(+\infty)=0 .\end{cases}
$$

Remark 1.1 The reaction term used here is more general than in [3], nevertheless, the results of [3] can be easily adapted to our case.

Remark 1.2 In the case $g(y)=y$ and $\Lambda \leq 1$ the solution $\left(u_{a d}, v_{a d}, c_{a d}\right)$ of (8-9) is shown to be unique (up to translation) in Marion [12]. Moreover, Bonnet [4] proved that uniqueness does not hold in the general case for $\Lambda>1$.

Let $\left(u_{s}, c_{s}\right)$ be the unique solution (see [3]) of the following adiabatic problem

$$
-\Lambda u_{s}^{\prime \prime}+c_{s} u_{s}^{\prime}=f\left(u_{s}, 1-u_{s}\right) \text { with } u_{s}(-\infty)=0, u_{s}(+\infty)=1 .
$$

Remark 1.3 Note that in the case $\Lambda=1$, the problem (8-9) reduces to the scalar case (10), and therefore, uniqueness always holds.

Under this hypothesis, we have the following results:

Theorem 1.4 1) For all $\Lambda>0$, if $\lambda$ is sufficiently small, there exist two distinct and nontrivial solutions $\left(u_{1}, v_{1}, c_{1}\right)$ and $\left(u_{2}, v_{2}, c_{2}\right)$ of the problem (1 -2), with $c_{1}<c_{2}$. Moreover, $v_{i}(i=1,2)$ is nonincreasing.

2) For all $\Lambda>0, c_{1} \rightarrow 0$ as $\lambda \rightarrow 0$. Moreover, if we assume that $\Lambda \leq 1$ and $g(y)=y$ on $\mathbb{R},\left(u_{2}, v_{2}, c_{2}\right)$ converges locally uniformly to $\left(u_{a d}, v_{a d}, c_{a d}\right)$, the unique solution of (8-9), as $\lambda \rightarrow 0$. In the case $\Lambda=1$, the same result holds for the reaction term $f(x, y)=p(x) g(y)$ satisfying $(4-6)$.

The next Theorem gives new bounds for every solution $(u, v, c)$ of (1-2).

Theorem 1.5 1) If $\lambda>\frac{f(1,1)}{h(\theta)}$, the problem (1-2) has no solution.

2) Assume that $g$ is globally Lipschitz continuous with constant $K$, then for all nontrivial solutions $(u, v, c)$ of (1-2),

$$
v(+\infty)>\exp \left(-\frac{K p(1)}{\lambda h(\theta)}\right) .
$$

3) Let $(u, v, c)$ be a solution of (1-2), then, for all $\Lambda \geq 1,0<c \leq c_{s}$, where $\left(u_{s}, c_{s}\right)$ is the solution of (10).

4) Let $(u, v, c)$ be a solution of (1-2), and set

$$
\begin{gathered}
\sigma_{1}=\max _{s \in(\theta, 1)} \frac{f(s, 1-s)}{s} \text { and } \sigma_{2}=\max _{s \in[0,1]} f(1-\underline{\Lambda} s, s) \text { with } \underline{\Lambda}:=\min \{\Lambda, 1\}, \text { then } \\
0<c<2 \sqrt{\sigma_{1} \Lambda} \text { for all } \Lambda \geq 1 \text { and } 0<c<\sqrt{\frac{\sigma_{2}}{\theta}} \text { for all } \Lambda>0 .
\end{gathered}
$$


Version définitive du manuscrit publié dans / Final version of the manuscript published in :

European Journal of Applied Mathematics, 2005, 16 (6), 741-765

\section{Proof of the existence theorem}

\subsection{Equivalence with a problem on $\mathbb{R}_{+}$}

In this section, we recall some results of Giovangigli. He establishes in [6, Proposition 2.2] that, under conditions (4) and (7),

Proposition 2.1 For all $c \geq 0$ and $\lambda \geq 0$, every solution (u,v) of (1-2), after a shift of the origin, is a solution of

$$
\left\{\begin{aligned}
-u^{\prime \prime}+c u^{\prime} & =f(u, v)-\lambda h(u) \quad \text { on }(0,+\infty), \\
-\Lambda v^{\prime \prime}+c v^{\prime} & =-f(u, v)
\end{aligned}\right.
$$

with the boundary conditions

$$
\left\{\begin{array}{l}
u(0)=\theta, \quad u^{\prime}(0)=c \theta+\lambda \int_{-\infty}^{0} h\left(u_{-}\right), v^{\prime}(0)=\frac{c}{\Lambda}(v(0)-1), \\
u(+\infty)=0, \quad v^{\prime}(+\infty)=0,
\end{array}\right.
$$

where $u_{-}$is the unique (see [6]) solution of

$$
\left\{\begin{array}{l}
-u_{-}^{\prime \prime}+c u_{-}^{\prime}+\lambda h\left(u_{-}\right)=0 \quad \text { on } \mathbb{R}_{-}, \\
u_{-}(-\infty)=0, u_{-}(0)=\theta .
\end{array}\right.
$$

Conversely, every solution $(u, v)$ of (11-13) can be extended to $\mathbb{R}$ in such a way that it is a nontrivial solution of (1-2).

Remark 2.2 This proposition clearly uses the fact that $p$ is of ignition type. It was demonstrated in [6] with $g(y)=y^{n}$ and a Lewis number $1 / \Lambda$ equal to 1 , but it is straightforwardly still valid with our more general reaction term $f(x, y)$ and with $1 / \Lambda \neq 1$.

Finally, Proposition (2.1) shows that solving (1-2) is equivalent to finding a solution $(u, v, c)$ of $(1)$ on $\mathbb{R}_{+}$with

$$
\begin{cases}u^{\prime}(0)=c u(0)+k(\theta, c, \lambda), & u(+\infty)=0, \quad u(0)=\theta, \\ \Lambda v^{\prime}(0)=c(v(0)-1), & v^{\prime}(+\infty)=0,\end{cases}
$$

where we have set $k(\theta, c, \lambda)=\lambda \int_{-\infty}^{0} h\left(u_{-}\right)$.

\subsection{Existence of solutions in bounded domains}

In order to use a topological degree argument, we study the system (1) on a bounded interval of $\mathbb{R}_{+}$. Namely, for each $a>0$, we set $I_{a}=(0, a)$, and we look for solutions $(u, v, c)$ in $C^{2}\left(I_{a},[0,1]\right)^{2} \times \mathbb{R}_{+}$of

$$
\left\{\begin{aligned}
-u^{\prime \prime}+c u^{\prime} & =f(u, v)-\lambda h(u) \\
-\Lambda v^{\prime \prime}+c v^{\prime} & =-f(u, v)
\end{aligned} \text { on } I_{a}\right.
$$


Version définitive du manuscrit publié dans / Final version of the manuscript published in :

European Journal of Applied Mathematics, 2005, 16 (6), 741-765

with the boundary conditions

$$
\left\{\begin{array}{lll}
u^{\prime}(0)=c u(0)+k(\theta, c, \lambda), & u(0)=\theta, & u(a)=0 \\
\Lambda v^{\prime}(0)=c(v(0)-1), & v(a)=0 .
\end{array}\right.
$$

Let us define the Banach space $X_{a}=C^{1}\left(I_{a},[0,1]\right) \times C^{1}\left(I_{a},[0,1]\right) \times \mathbb{R}$, with the norm $\|(u, v, c)\|_{X_{a}}=\|u\|_{C^{1}\left(I_{a}\right)}+\|v\|_{C^{1}\left(I_{a}\right)}+|c|$, and let $J_{\tau}$ be the mapping defined by

$$
J_{\tau}: X_{a} \longrightarrow X_{a},(u, v, c) \mapsto(U, V, c+\theta-U(0)),
$$

where $(U, V)$ is the unique solution of the linear problem

$$
\left\{\begin{aligned}
-U^{\prime \prime}+c U^{\prime} & =\tau[f(u, v)-\lambda h(u)]+(1-\tau)(V-\lambda U) \\
-\Lambda_{\tau} V^{\prime \prime}+c V^{\prime} & =-\tau f(u, v)-(1-\tau) V
\end{aligned}\right.
$$

on $I_{a}=(0, a)$ with the boundary conditions,

$$
\begin{cases}U^{\prime}(0)=c U(0)+\tau k(\theta, c, \lambda), & U(a)=0 \\ \Lambda_{\tau} V^{\prime}(0)=c V(0)-c, & V(a)=0\end{cases}
$$

where $\Lambda_{\tau}=\tau \Lambda+(1-\tau)$.

Remark 2.3 This mapping $J_{\tau}$ is close to that of [6]. The homotopic transformation of [3] cannot be used here. Indeed, we will see in the proof of Proposition 2.10 that the right-hand side plays a crucial role in the definition of a positive real number $c_{*}$ such that no solution $(u, v, c)$ exists with $c \in\left(\varepsilon, c_{*}-\varepsilon\right)$ for $\lambda$ small enough (and $\varepsilon$ arbitrarily small).

Let $\left(u_{\tau}, v_{\tau}, c_{\tau}\right)$ be a fixed point of the mapping $J_{\tau}$, with $\tau \in[0,1]$. In order to compute a topological degree, we need some a priori estimates on $\left(u_{\tau}, v_{\tau}, c_{\tau}\right)$. More precisely, we prove the following proposition:

Proposition 2.4 Let $\left(u_{\tau}, v_{\tau}, c_{\tau}\right)$ be a fixed point of the mapping $J_{\tau}$, with $c \geq 0$. Then

$$
\left\{\begin{array}{lcl}
0<u_{\tau}<1, & 0<v_{\tau}<1, & v_{\tau} \leq \frac{1-u_{\tau}}{\min \left\{1, \Lambda_{\tau}\right\}}, \\
-c_{\tau}<u_{\tau}^{\prime}<c_{\tau}\left(1+\frac{1}{\Lambda_{\tau}}\right), & -\frac{c_{\tau}}{\Lambda_{\tau}}<v_{\tau}^{\prime}<0, & 0<c_{\tau} \leq \sqrt{\frac{f(1,1)+1}{\theta}} .
\end{array}\right.
$$

Proof of Proposition 2.4: For the readability of the following calculations, we will shall omit the index $\tau$ by replacing $\left(u_{\tau}, v_{\tau}, c_{\tau}\right)$ with $(u, v, c)$.

Lemma $2.5 v>0$ on $I_{a}$.

Proof of Lemma 2.5 For each $\tau \in(0,1), v$ satisfies the following equation

$$
-\Lambda_{\tau} v^{\prime \prime}+c v^{\prime}+\gamma v=0 \text { on } I_{a},
$$




\section{Version définitive du manuscrit publié dans / Final version of the manuscript} published in :

European Journal of Applied Mathematics, 2005, 16 (6), 741-765

where we have set $\gamma(x):=\frac{\tau f(u, v)+(1-\tau) v}{v}$ if $v(x) \neq 0$, and $\gamma(x):=1$ if $v(x)=0$.

From our hypothesis (3-6), we have $\gamma(x) \geq 0$ on $I_{a}$. Therefore, using a maximum principle (see e.g. [5]), we obtain $\inf _{x \in I_{a}} v(x)=\min \{v(0), v(a)\}$. Let us assume that $v(0)<0$, then, from $(18), v^{\prime}(0) \leq 0$. Since $v(a)=0$, we necessarily have $v^{\prime}(0)=0$, which implies $c=0$ from (18). Therefore, from (20), $v$ is concave as long as $v(x) \leq 0$, which is impossible since $v(a)=0$. It follows that $v \geq 0$. From the strong maximum principle applied to (20), we have $v>0$ on $I_{a}$.

Lemma $2.6 v<1$ and $-\frac{c}{\Lambda_{\tau}}<v^{\prime}<0$ on $I_{a}$.

Proof of Lemma 2.6: From (20), and since $v>0$, we have $\left(e^{-\frac{c}{\Lambda_{\tau}} x} v^{\prime}\right)^{\prime} \geq 0$ on $I_{a}$.

Thus, integrating between $x \in I_{a}$ and $a$, we obtain $e^{-\frac{c}{\Lambda_{\tau}}(a-x)} v^{\prime}(a) \geq v^{\prime}(x)$. Since $v>0$ on $I_{a}$ and $v(a)=0$, it is then classical that $v^{\prime}<0$. It then follows from (18) that $v(0)<1$, hence $v<1$ on $I_{a}$. Furthermore, from $(20), \Lambda_{\tau} v^{\prime}-c v$ is nondecreasing on $I_{a}$, thus

$$
v^{\prime}(x)>-\frac{c}{\Lambda_{\tau}} \text { on } I_{a},
$$

from (18), and since $v>0$

Lemma 2.7 $0<u<1$ and $v \leq \frac{1-u}{\min \left\{1, \Lambda_{\tau}\right\}}$ on $I_{a}$.

Proof of Lemma 2.7: From the strong maximum principle, and using the hypothesis (7), we easily obtain $u>0$ on $I_{a}$. Moreover, setting $w=u+v$, and integrating the equation satisfied by $w$ between 0 and $x \in I_{a}$, we obtain, using (18), and since $h(u) \geq 0,-w^{\prime}(x)+c w(x)-c+k \leq\left(\Lambda_{\tau}-1\right) v^{\prime}(x)$ on $I_{a}$. Thus, since $k \geq 0,-\left[(w-1) e^{-c x}\right]^{\prime} \leq e^{-c x}\left(\Lambda_{\tau}-1\right) v^{\prime}(x)$ on $I_{a}$, and integrating between $x$ and $a$ gives

$$
w(x) \leq\left(\Lambda_{\tau}-1\right) e^{c x} \int_{x}^{a} v^{\prime}(t) e^{-c t} d t+1 .
$$

As a consequence, if $\Lambda_{\tau}>1$, we have $u+v \leq 1$ since $v^{\prime} \leq 0$ from the above calculations. Next, if $\Lambda_{\tau} \leq 1$, we deduce from (22) that $w \leq\left(1-\Lambda_{\tau}\right) v+1$. Therefore $u \leq 1-\Lambda_{\tau} v$. Thus, since $v>0$, in both cases $u<1$. Also, we see that $v \leq \frac{1-u}{\min \left\{1, \Lambda_{\tau}\right\}}$.

Lemma $2.8-c<u^{\prime}<c\left(1+\frac{1}{\Lambda_{\tau}}\right)$ on $I_{a}$.

Proof of Lemma 2.8: Let us add the equations satisfied by $u$ and $v$, and integrate the sum between 0 and $x$. Using (21) and the boundary conditions (18), we obtain $u^{\prime}>-c$. Moreover, setting $y=u+\Lambda_{\tau} v$ and using arguments similar to those in Lemma 2.7, we get $u^{\prime}<c\left(1+\frac{1}{\Lambda_{\tau}}\right)$. 
Version définitive du manuscrit publié dans / Final version of the manuscript published in :

European Journal of Applied Mathematics, 2005, 16 (6), 741-765

Lemma $2.90<c \leq \sqrt{\frac{f(1,1)+1}{\theta}}$.

Proof of Lemma 2.9: The proof of the inequality $c>0$ is standard (see e.g. [3]). The upper bound for $c$ follows from a simple comparison principle argument.

Proposition (2.4) is proved.

In order to compute a topological degree, we need another estimate that will be established for small parameters $\lambda$.

Let $\left(u_{\tau}, v_{\tau}, c_{\tau}\right) \in X_{a}$ be a fixed point of $J_{\tau}$.

Proposition 2.10 For all $\varepsilon>0$, there exist $\lambda_{1}>0$ and $a_{1}>0$ such that for all $a>a_{1}$ and for all $\tau \in[0,1]$,

$$
\left(\lambda<\lambda_{1}\right) \Longrightarrow\left(c_{\tau} \notin\left(\varepsilon, c_{*}-\varepsilon\right)\right),
$$

where $c_{*}$ is a positive real number defined at the end of the proof.

Proof of Proposition 2.10: Let $\left(u_{n}, v_{n}, c_{n}, \tau_{n}\right)$ be a sequence of fixed points of $J_{\tau_{n}}$, with $c_{n} \in\left(\varepsilon, c_{*}-\varepsilon\right), \tau_{n} \in[0,1], a_{n} \rightarrow+\infty, \lambda_{n} \rightarrow 0$ and $\varepsilon>$ 0 . Then, from Proposition 2.4, the sequence $\left(u_{n}, v_{n}, c_{n}, \tau_{n}\right)_{n \in \mathbb{N}}$ is bounded in $\left[C^{2}\left(0, a_{n}\right)\right]^{2} \times \mathbb{R} \times[0,1]$. By compactness, we obtain the convergence (up to the extraction of some subsequence), in $C_{\text {loc }}^{1}\left(\mathbb{R}_{+}\right)^{2} \times \mathbb{R} \times[0,1]$ of $\left(u_{n}, v_{n}, c_{n}, \tau_{n}\right)$ to $(u, v, c, \tau)$ which is a classical solution of

$$
\left\{\begin{aligned}
-u^{\prime \prime}+c u^{\prime} & =\tau f(u, v)+(1-\tau) v \\
-\Lambda_{\tau} v^{\prime \prime}+c v^{\prime} & =-\tau f(u, v)-(1-\tau) v
\end{aligned} \text { on } \mathbb{R}_{+},\right.
$$

with

$$
u^{\prime}(0)=c \theta, \Lambda_{\tau} v^{\prime}(0)=c v(0)-c, u(0)=\theta,
$$

since $k(\theta, c, 0)=0$ (see (18)) and

$$
c \in\left[\varepsilon, c_{*}-\varepsilon\right] .
$$

Let us set $\Lambda_{*}=\min \left\{1, \frac{1}{\Lambda_{\tau}}\right\}$ and $\Lambda^{*}=\max \left\{1, \frac{1}{\Lambda_{\tau}}\right\}$. We have the following:

Lemma $2.11 \Lambda_{*}(1-u) \leq v \leq \Lambda^{*}(1-u)$.

Proof of Lemma 2.11: $v \leq \Lambda^{*}(1-u)$ is a consequence of Proposition (2.4). Setting $w=u+v$ and $y=u+\Lambda_{\tau} v$, a straightforward computation leads to

$$
\left\{\begin{array}{l}
w(x)=1+\left(\Lambda_{\tau}-1\right) e^{c x} \int_{x}^{+\infty} v^{\prime}(t) e^{-c t} d t \\
y(x)=1+c\left(\Lambda_{\tau}-1\right) e^{c x} \int_{x}^{+\infty} v(t) e^{-c t} d t
\end{array} \text { on } \mathbb{R}_{+},\right.
$$

thus

$$
\left\{\begin{array}{l}
v(x)=1-u(x)+\left(\Lambda_{\tau}-1\right) e^{c x} \int_{x}^{+\infty} v^{\prime}(t) e^{-c t} d t \\
v(x)=\frac{1-u(x)}{\Lambda_{\tau}}+\frac{\left(\Lambda_{\tau}-1\right)}{\Lambda_{\tau}} e^{c x} \int_{x}^{+\infty} v(t) e^{-c t} d t
\end{array} \text { on } \mathbb{R}_{+} .\right.
$$


Version définitive du manuscrit publié dans / Final version of the manuscript published in :

European Journal of Applied Mathematics, 2005, 16 (6), 741-765

Therefore, if $\Lambda_{\tau} \leq 1$, then $v \geq 1-u$ since $v^{\prime} \leq 0$, and if $\Lambda>1$, then $v \geq \frac{1-u}{\Lambda_{\tau}}$ since $v>0$. That concludes the proof of the lemma.

Lemma 2.12 The function $u+v-1$ has a constant sign on $\mathbb{R}_{+}$.

Proof of Lemma 2.12: From Lemma 2.11, $(1-u)\left(\Lambda_{*}-1\right) \leq u+v-1 \leq$ $(1-u)\left(\Lambda^{*}-1\right)$. Thus, if $\Lambda_{\tau}>1, \Lambda^{*}=1$ and $u+v-1 \leq 0$, and if $\Lambda_{\tau} \leq 1$, $\Lambda_{*}=1$ and we get $u+v-1 \geq 0$.

Lemma $2.13 u^{\prime}(+\infty)=v^{\prime}(+\infty)=u^{\prime \prime}(+\infty)=v^{\prime \prime}(+\infty)=0$.

Proof of Lemma 2.13: Let us integrate the equation satisfied by $v$ from 0 to $x \in \mathbb{R}_{+}$. We obtain

$$
\Lambda_{\tau} v^{\prime}(x)-c v(x)=-c+\int_{0}^{x}[\tau f(u, v)+(1-\tau) v(x)] d x .
$$

Since $v$ is nonincreasing and nonnegative, it has got a finite limit as $x \rightarrow+\infty$. Furthermore, since $\tau f(u, v)+(1-\tau) v(x) \geq 0$, the integral $\int_{0}^{x}[\tau f(u, v)+(1-\tau) v(x)] d x$ converges (even to $+\infty$ ). As a consequence, from (26), $v^{\prime}(x)$ has a limit as $x \rightarrow+\infty$. Since $v$ is bounded, this limit is equal to 0 . Integrating the equation satisfied by $y=u+\Lambda_{\tau} v$ from 0 to $x \in \mathbb{R}_{+}$, we obtain

$$
\Lambda_{\tau} v^{\prime}(x)+u^{\prime}(x)=c(u(x)+v(x)-1) .
$$

Therefore $\Lambda_{\tau} v(x)+u(x)=c \int_{0}^{x}(u(t)+v(t)-1) d t+\Lambda_{\tau} v(0)+\theta$. But, from Lemma 2.12 the integral of the right-hand side converges, thus $u$ admits a limit at $+\infty$. Arguing as for $v$ we deduce that $u^{\prime}(+\infty)=0$. It then follows from (23) that $u^{\prime \prime}(+\infty)=v^{\prime \prime}(+\infty)=0$. That completes the proof of Lemma 2.13.

Lemma $2.14 u^{\prime} \geq 0$ on $\mathbb{R}_{+}$

Proof of Lemma 2.14: The equation satisfied by $u$ in (23) is equivalent to

$$
-\left(u^{\prime}(x) e^{-c x}\right)^{\prime}=e^{-c x}[\tau f(u(x), v(x))+(1-\tau) v(x)] \geq 0 .
$$

Therefore, integrating this expression from $x \in \mathbb{R}_{+}$to $+\infty$, and using Lemma 2.13 we obtain the sought result.

Lemma $2.15 u(+\infty)=1$ and $v(+\infty)=0$.

Proof of Lemma 2.15: From (23) and (27), $u(+\infty)$ and $v(+\infty)$ satisfy

$$
\left\{\begin{array}{l}
\tau f[u(+\infty), v(+\infty)]+v(+\infty)(1-\tau)=0 \\
u(+\infty)+v(+\infty)=1
\end{array}\right.
$$

Thus, since $c>\varepsilon$, we have $u^{\prime}(0)=c \theta>0$. Therefore, from Lemma 2.14, $u(+\infty)>\theta$. It follows that $v(+\infty)=0$ and $u(+\infty)=1$. 


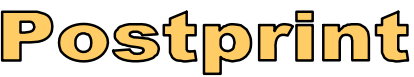

Version définitive du manuscrit publié dans / Final version of the manuscript published in :

European Journal of Applied Mathematics, 2005, 16 (6), 741-765

Using Lemma 2.11, we obtain:

$$
\begin{aligned}
-u^{\prime \prime}+c u^{\prime} & =\tau f(u, v)+(1-\tau) v \\
& \leq \tau f\left(u, \Lambda^{*}(1-u)\right)+(1-\tau) \Lambda^{*}(1-u) .
\end{aligned}
$$

Moreover, from the hypothesis (6) and from the definition of $k^{*}\left(\Lambda^{*}\right)$, we see that $f\left(u, \Lambda^{*}(1-u)\right) \leq k^{*}\left(\Lambda^{*}\right) f(u, 1-u)$, and

$$
\Lambda^{*} \leq k^{*}\left(\Lambda^{*}\right)<+\infty,
$$

hence, from (29) and (30),

$$
-u^{\prime \prime}+c u^{\prime} \leq k^{*}\left(\Lambda^{*}\right)[\tau f(u, 1-u)+(1-\tau)(1-u)] .
$$

Let us now multiply the inequality $(31)$ by $u$ and integrate over $(0,+\infty)$. We then obtain, using again Lemma 2.13,

$$
\int_{0}^{\infty}\left(u^{\prime}\right)^{2}+\frac{c}{2}\left(1+\theta^{2}\right) \leq k^{*}\left(\Lambda^{*}\right) \int_{0}^{\infty}[\tau f(u, 1-u)+(1-\tau)(1-u)],
$$

since $u \leq 1$. Next, still using Lemma 2.11, we get

$$
\begin{aligned}
-u^{\prime \prime}+c u^{\prime} & =\tau f(u, v)+(1-\tau) v \\
& \geq \tau f\left(u, \Lambda_{*}(1-u)\right)+(1-\tau) \Lambda_{*}(1-u) .
\end{aligned}
$$

Again, from the definition of $k_{*}\left(\Lambda_{*}\right)$, we have $f\left(u, \Lambda_{*}(1-u)\right) \geq k_{*}\left(\Lambda_{*}\right) f(u, 1-u)$ and

$$
0<k_{*}\left(\Lambda_{*}\right) \leq \Lambda_{*} .
$$

Then, integrating (33) over $(0,+\infty)$, and using (34), we get, from the limiting behaviours obtained in the previous lemmas,

$$
c \geq k_{*}\left(\Lambda_{*}\right) \int_{0}^{\infty}[\tau f(u, 1-u)+(1-\tau)(1-u)],
$$

thus, with (32), we obtain the inequality

$$
\int_{0}^{\infty}\left(u^{\prime}\right)^{2}+\frac{c}{2}\left(1+\theta^{2}\right) \leq \frac{k^{*}\left(\Lambda^{*}\right)}{k_{*}\left(\Lambda_{*}\right)} c,
$$

which is equivalent to

$$
\int_{0}^{\infty}\left(u^{\prime}\right)^{2} \leq \frac{c}{2}\left(2 \frac{k^{*}\left(\Lambda^{*}\right)}{k_{*}\left(\Lambda_{*}\right)}-1-\theta^{2}\right) .
$$

Next, let us multiply the equality (28) by $u^{\prime}$ and integrate it over $(0,+\infty)$. Using again Lemma 2.11 and the result (34) above, we get

$$
\frac{\theta^{2} c^{2}}{2}+c \int_{0}^{\infty}\left(u^{\prime}\right)^{2} \geq k_{*}\left(\Lambda_{*}\right) \int_{0}^{\infty} u^{\prime}[\tau f(u, 1-u)+(1-\tau)(1-u)],
$$


Version définitive du manuscrit publié dans / Final version of the manuscript published in :

European Journal of Applied Mathematics, 2005, 16 (6), 741-765

which gives, using Lemma 2.14 and inequality (35),

$$
\begin{aligned}
\frac{c^{2}}{2}\left(2 \frac{k^{*}\left(\Lambda^{*}\right)}{k_{*}\left(\Lambda_{*}\right)}-1\right) & \geq k_{*}\left(\Lambda_{*}\right) \int_{\theta}^{1}[\tau f(s, 1-s)+(1-\tau)(1-s)] d s \\
& \geq k_{*}\left(\Lambda_{*}\right) m_{*},
\end{aligned}
$$

where $m_{*}$ is defined by $m_{*}=\min \left\{m_{1}, m_{2}\right\}$, with

$$
m_{1}:=\int_{\theta}^{1} f(s, 1-s) d s \text { and } m_{2}=\int_{\theta}^{1}(1-s) d s .
$$

Therefore,

$$
c^{2} \geq 2 \frac{k_{*}\left(\Lambda_{*}\right)^{2}}{2 k^{*}\left(\Lambda^{*}\right)-k_{*}\left(\Lambda_{*}\right)} m_{*}>0 \text { from (30) and (34), }
$$

thus, if $\Lambda<1$ then $\Lambda_{*}=1$ and $k_{*}\left(\Lambda_{*}\right)=1$. Furthermore, $\Lambda^{*} \leq \frac{1}{\Lambda}$, which implies from the definition of $k^{*}$ that $k^{*}\left(\Lambda^{*}\right) \leq k^{*}\left(\frac{1}{\Lambda}\right)$. Similarly, for $\Lambda \geq 1, \Lambda^{*}=1$ and $k^{*}\left(\Lambda^{*}\right)=1$. Furthermore, $\Lambda_{*} \geq \frac{1}{\Lambda}$, which implies that $k_{*}\left(\Lambda_{*}\right) \geq k_{*}\left(\frac{1}{\Lambda}\right)$. Also, it follows from (6) that $\frac{1}{\Lambda} \leq k^{*}\left(\frac{1}{\Lambda}\right)<+\infty$ and $0<k_{*}\left(\frac{1}{\Lambda}\right) \leq \frac{1}{\Lambda}$. Thus we can set

$$
c_{*}:=\sqrt{\frac{2}{2 k^{*}\left(\frac{1}{\Lambda}\right)-1} m_{*}} \text { if } \Lambda<1 \text { and } c_{*}:=\sqrt{\frac{2 k_{*}\left(\frac{1}{\Lambda}\right)^{2}}{2-k_{*}\left(\frac{1}{\Lambda}\right)} m_{*}} \text { if } \Lambda \geq 1 .
$$

Then $c_{*}$ is positive and independent of $\tau$ (although $\Lambda^{*}$ and $\Lambda_{*}$ depend on $\tau$ ). Moreover, we have $c \geq c_{*}$, which is in contradiction to (25). That completes the proof of Proposition 2.10.

In order to compute a topological degree, we need to investigate the case $\tau=0 .(u, v, c)$ is a fixed point of $J_{0}$ in $X_{a}$ if and only if it satisfies

$$
\left\{\begin{array}{ll}
-u^{\prime \prime}+c u^{\prime}+\lambda u=v \\
-v^{\prime \prime}+c v^{\prime}+v=0
\end{array} \text { in } I_{a}, \begin{cases}u^{\prime}(0)=c u(0), & u(a)=0, \quad u(0)=\theta, \\
v^{\prime}(0)=c v(0)-c, & v(a)=0 .\end{cases}\right.
$$

Proposition 2.16 For $\lambda$ small enough and a large enough, (37) admits exactly two solutions $\left(u^{1}, v^{1}, c^{1}\right)$ and $\left(u^{2}, v^{2}, c^{2}\right)$. Moreover, there exists $c_{\theta}>0$, such that for all $\varepsilon>0, \exists \lambda_{2}>0$ and $a_{2}>0$, such that for all $\lambda<\lambda_{2}$ and for all $a>a_{2}, 0<c^{1}<\varepsilon$ and $c^{2}>c_{\theta}$.

Proof of Proposition 2.16: For each $c$ there is only one pair of functions $(u, v)$ which satisfies (37). We can compute these functions explicitly. Let us set $\phi(c):=u(0)(c)$. In order to solve the equation $\phi(c)=\theta$, we study the 
Version définitive du manuscrit publié dans / Final version of the manuscript published in :

European Journal of Applied Mathematics, 2005, 16 (6), 741-765

function $c \mapsto \phi(c)$ on $\mathbb{R}_{+}$. The results of the Appendix show that for $\lambda$ small enough and $a$ large enough, the equation $\phi(c)=\theta$ admits exactly two solutions $c^{1}$ and $c^{2}$, with $0<c^{1}<(2 \lambda)^{1 / 3}$ and $c^{2}>c_{\theta}$, where $c_{\theta}$ is positive and does not depend on $\lambda$ and $a$. The proof of Proposition 2.16 is then complete.

Using Propositions 2.4, 2.10 and 2.16, we are now able to define topological degrees. First, set $K_{\tau} \equiv I-J_{\tau}$, where $I$ is the identity mapping of $X_{a}$. Coming back to the definition of $J_{\tau}$, with (17) and (18), we can easily check that $U$ and $V$ are bounded in $C^{2}\left(I_{a}\right)$, if $u$ and $v$ are in $C^{1}\left(I_{a}\right)$. Furthermore $C^{2}\left(I_{a}\right)$ is compactly embedded into $C^{1}\left(I_{a}\right)$. Therefore the mappings $J_{\tau}$ and $K_{\tau}$ are compact. Similarly, the mapping $F: X_{a} \times[0,1] \rightarrow\left(X_{a} \mapsto X_{a}\right)$ defined by $(u, v, c, \tau) \mapsto K_{\tau}$ is compact and uniformly continuous with respect to $\tau$. Moreover, we notice that $(u, v, c)$ is a classical solution of (15-16) if and only if $K_{1}(u, v, c)=0$, therefore, we look for the solutions of this equation. From the properties of $K_{\tau}$, and using the homotopy invariance of the topological degree (see [14]), we only need to compute the degree for $\tau=0$. First let us prove that a degree can be defined. Set $\underline{\Lambda}:=\min \{\Lambda, 1\}, M:=f(1,1)+1$ and

$$
\Omega=\left\{(u, v, c) \in X_{a}:\|(u, v, c)\|_{\left(X_{a}\right)}<2+2 \sqrt{\frac{M}{\theta}}\left(1+\frac{1}{\underline{\Lambda}}\right)\right\} .
$$

From Proposition 2.4, we know that

Proposition 2.17 For all $\tau \in[0,1]$, and for a large enough,

$$
0 \notin K_{\tau}(\partial \Omega) .
$$

Next, we define two open sets of $X_{a}$, in which the topological degree will be computed. Let us set $\varepsilon>0$ such that $\varepsilon<\min \left\{\frac{c_{*}}{8}, c_{\theta}\right\}$ where $c_{*}$ and $c_{\theta}$ are defined in Propositions 2.10 and 2.16 respectively, $a_{*}:=\max \left\{a_{1}, a_{2}\right\}$ and $\lambda_{*}:=\min \left\{\lambda_{1}, \lambda_{2}\right\}$, where $a_{1}, a_{2}, \lambda_{1}$ and $\lambda_{2}$ are defined in Propositions 2.10 and 2.16. Now, set

$$
\begin{aligned}
& O_{1}^{a}:=\Omega \cap\left\{(u, v, c) \in X_{a} \text { s.t. } c<2 \varepsilon\right\}, \text { and } \\
& O_{2}^{a}:=\Omega \cap\left\{(u, v, c) \in X_{a} \text { s.t. } c>c_{*}-2 \varepsilon\right\} .
\end{aligned}
$$

Notice that $O_{2}^{a} \neq\{\emptyset\}$ : since $\varepsilon<c_{\theta}$, we deduce from Propositions 2.10 and 2.16 that $c^{2}>c_{*}-\varepsilon\left(c^{2}\right.$ is defined in Proposition 2.16), thus Proposition 2.4 ensures that

$$
\sqrt{\frac{M}{\theta}}>c^{2}>c_{*}-\varepsilon>c_{*}-2 \varepsilon .
$$

We have the following

Proposition 2.18 For all $\lambda<\lambda_{*}$, for all $\tau \in[0,1], 0 \notin K_{\tau}\left(\partial O_{i}^{a}\right)$, for $i=1,2$, and for $a \geq a_{*}$. 
Version définitive du manuscrit publié dans / Final version of the manuscript published in :

European Journal of Applied Mathematics, 2005, 16 (6), 741-765

Proof of Proposition 2.18: Let $\left(u_{i}^{*}, v_{i}^{*}, c_{i}^{*}\right)$ be a solution of $K_{\tau}=0$ in $\partial O_{i}^{a}$ $(i=1,2)$. Then we deduce from Proposition 2.17 that $c_{1}^{*}=2 \varepsilon\left(\right.$ and $\left.c_{2}^{*}=c_{*}-2 \varepsilon\right)$. From Proposition 2.10, $c_{i}^{*} \notin\left(\varepsilon, c_{*}-\varepsilon\right)(i=1,2)$, thus we get a contradiction, and the proposition is proved.

Proposition 2.18 enables us to define the Leray-Schauder degree $\operatorname{deg}\left(K_{\tau}, O_{i}^{a}, 0\right)$, for $i=1,2$. Let us compute its value:

Proposition 2.19 Let us assume that $\lambda<\lambda_{*}$ and $a \geq a_{*}$; for $i=1,2$ we have $\operatorname{deg}\left(K_{1}, O_{1}^{a}, 0\right)=\operatorname{deg}\left(K_{0}, O_{1}^{a}, 0\right)=1$ and $\operatorname{deg}\left(K_{1}, O_{2}^{a}, 0\right)=\operatorname{deg}\left(K_{0}, O_{2}^{a}, 0\right)=-1$.

Proof of Proposition 2.19: From the compactness of the mapping $K_{\tau}$, and its uniform continuity with respect to $\tau$, we obtain, using the homotopic invariance of the Leray-Schauder degree (see [14]),

$$
\operatorname{deg}\left(K_{1}, O_{i}^{a}, 0\right)=\operatorname{deg}\left(K_{0}, O_{i}^{a}, 0\right) .
$$

Furthermore, $K_{0}$ is known explicitly:

$$
K_{0}: X_{a} \rightarrow X_{a}:(u, v, c) \mapsto\left(u-U_{0}(c), v-V_{0}(c), U_{0}(0)-\theta\right)
$$

where $U_{0}$ and $V_{0}$ are the solutions of (17-18). Moreover, this mapping is homotopic to

$$
\Phi_{\tau}: X_{a} \rightarrow X_{a}:(u, v, c) \mapsto\left(u-\tau U_{0}(c), v-\tau V_{0}(c), U_{0}(0)-\theta\right) .
$$

Besides, since $\lambda<\lambda_{*} \leq \lambda_{2}, c^{1}<\varepsilon$ and, as noted in (41), $c^{2}>c_{*}-\varepsilon>c_{*}-2 \varepsilon$ ( $c^{1}$ and $c^{2}$ are defined in Proposition 2.16). Hence, defining $\phi$ as in the proof of Proposition 2.16, the equation $\phi(c)=\theta$ admits exactly one solution in $(0,2 \varepsilon)$ and one solution in $\left(c_{*}-2 \varepsilon, \sqrt{\frac{M}{\theta}}\right)$. Using the homotopy invariance and the multiplicative properties of the degree, we find that $\operatorname{deg}\left(K_{1}, O_{1}^{a}, 0\right)=\operatorname{deg}\left(\Phi_{0}, O_{1}^{a}, 0\right)=$ $\operatorname{deg}(\phi(c)-\theta,(0,2 \varepsilon), 0)=1$ and $\operatorname{deg}\left(K_{1}, O_{2}^{a}, 0\right)=\operatorname{deg}\left(\Phi_{0}, O_{2}^{a}, 0\right)=\operatorname{deg}(\phi(c)-$ $\left.\theta,\left(c_{*}-2 \varepsilon, \sqrt{\frac{M}{\theta}}\right), 0\right)=-1$ for $a \geq a_{*}$ (the sign of the degree is given by the sign of $\phi^{\prime}$, see [6, Proposition 4.6]).

Remark 2.20 The real $\varepsilon$ can be chosen as small as we want, provided $\lambda$ is sufficiently small.

As a consequence of Proposition 2.19, it follows that:

Corollary 2.21 For $\lambda<\lambda_{*}$, and for $a>a_{*}$, the problem (15-16) admits at least two classical solutions $\left(u_{1}^{a}, v_{1}^{a}, c_{1}^{a}\right)$ and $\left(u_{2}^{a}, v_{2}^{a}, c_{2}^{a}\right)$, with $0<c_{1}^{a}<\frac{c_{*}}{4}$ and $\frac{3 c_{*}}{4}<c_{2}^{a}$. Moreover, from Remark 2.20, we can assume that $c_{1}^{a}<r_{\lambda}$, where $r_{\lambda}$ does not depend on a and $r_{\lambda} \rightarrow 0$ as $\lambda \rightarrow 0$. 
Version définitive du manuscrit publié dans / Final version of the manuscript published in :

European Journal of Applied Mathematics, 2005, 16 (6), 741-765

\subsection{Passage to the limit in $\mathbb{R}_{+}$}

For $\lambda<\lambda_{*}$, and for a large enough, let $\left(u_{1}^{a}, v_{1}^{a}, c_{1}^{a}\right)$ and $\left(u_{2}^{a}, v_{2}^{a}, c_{2}^{a}\right)$ be the solutions obtained in Corollary 2.21. Using Proposition 2.4, we find that, for $a$ large enough, $\left(u_{1}^{a}, v_{1}^{a}, c_{1}^{a}\right)$ and $\left(u_{2}^{a}, v_{2}^{a}, c_{2}^{a}\right)$ are bounded (independently of $a$ ) in $C^{2}\left(I_{a}\right) \times C^{2}\left(I_{a}\right) \times \mathbb{R}_{+}$. By compactness, there exist two sequences $\left(a_{n}^{1}\right)_{n \in \mathbb{N}}$ and $\left(a_{n}^{2}\right)_{n \in \mathbb{N}}$ such that $\left(u_{1}^{a_{n}^{1}}, v_{1}^{a_{n}^{1}}, c_{1}^{a_{n}^{1}}\right)$ and $\left(u_{2}^{a_{n}^{2}}, v_{2}^{a_{n}^{2}}, c_{2}^{a_{n}^{2}}\right)$ converge in $C_{l o c}^{1}\left(\mathbb{R}_{+}\right) \times$ $C_{l o c}^{1}\left(\mathbb{R}_{+}\right) \times \mathbb{R}_{+}$to $\left(u_{1}, v_{1}, c_{1}\right)$ and $\left(u_{2}, v_{2}, c_{2}\right)$ respectively. When the distinction between the two solutions is not needed, we may use the general notation $(u, v, c)$ for $\left(u_{1}, v_{1}, c_{1}\right)$ and $\left(u_{2}, v_{2}, c_{2}\right)$ henceforth. $(u, v, c)$ satisfies:

$$
\left\{\begin{array}{l}
-u^{\prime \prime}+c u^{\prime}=f(u, v)-\lambda h(u) \\
-\Lambda v^{\prime \prime}+c v^{\prime}=-f(u, v)
\end{array} \quad \text { on } \mathbb{R}\right.
$$

with the boundary conditions,

$$
u^{\prime}(0)=c u(0)+k(\theta, c, \lambda), u(0)=\theta, \Lambda v^{\prime}(0)=c v(0)-c .
$$

Moreover, since the real numbers $\lambda_{*}, c_{*}$ and $r_{\lambda}$ of Corollary 2.21 are independent of $a$, we see that

$$
0 \leq c_{1} \leq r_{\lambda} \text { and } \frac{3 c_{*}}{4} \leq c_{2} .
$$

Now let us prove that:

Lemma 2.22 The two solutions found above satisfy

$$
u(+\infty)=0, u^{\prime}(+\infty)=v^{\prime}(+\infty)=0 \text { and } u^{\prime \prime}(+\infty)=v^{\prime \prime}(+\infty)=0 .
$$

Proof of Lemma 2.22: Passing to the limit $a \rightarrow+\infty$ in Proposition 2.4, we find that $v$ is nonnegative and nonincreasing. Let us integrate the equation satisfied by $v$ in (42) from 0 to $x>0$. We obtain

$$
\Lambda v^{\prime}(x)-c v(x)=\Lambda v^{\prime}(0)-c v(0)+\int_{0}^{x} f(u, v)(s) d s .
$$

Therefore, since $f(u, v) \geq 0$ on $\mathbb{R}_{+}$, the right-hand side of the above equation converges as $x \rightarrow+\infty$. Hence $v^{\prime}(+\infty)$ is defined. Since $v$ is bounded, $v^{\prime}(+\infty)=$ 0 .

Next, let us add the equations satisfied by $u$ and $v$ in (42). This gives, after an integration from 0 to $x>0$ :

$$
\begin{gathered}
-\Lambda v^{\prime}(x)-u^{\prime}(x)+c u(x)+c v(x)= \\
-\Lambda v^{\prime}(0)+u^{\prime}(0)-c u(0)-c v(0)-\lambda \int_{0}^{x} h(u)(s) d s .
\end{gathered}
$$

Since the left-hand side is bounded, and since $h(u)$ is nonnegative (because $u \geq 0$ ), we get $\lim _{x \rightarrow+\infty} h(u)(x)=0$. From the hypothesis (7) on $h$, it follows 
Version définitive du manuscrit publié dans / Final version of the manuscript published in :

European Journal of Applied Mathematics, 2005, 16 (6), 741-765

that $u(+\infty)=0$. Now, using the above results on $v^{\prime}(+\infty)$, equation (47) implies that $u^{\prime}(+\infty)$ exits. Since $u$ is bounded, we find $u^{\prime}(+\infty)=0$. Then $u^{\prime \prime}(+\infty)=v^{\prime \prime}(+\infty)=0$ immediately follows from $(42)$.

From Proposition 2.1, we have found two distinct solutions of (1-2). In order to complete the proof of Theorem 1.4, it only remains to prove that these two solutions are nontrivial. Since $u(0)=\theta$ and $u(+\infty)=0, u$ is not a trivial solution. Let us assume that $v$ is a constant. Then it follows from (42) that $f(u, v)=0$ on $\mathbb{R}$, hence $-u^{\prime \prime}+c u^{\prime}+\lambda h(u)=0$ on $\mathbb{R}$. Since $u( \pm \infty)=0$, we obtain that $u \equiv 0$ from the maximum principle, which contradicts $u(0)=\theta$.

Part 1) of Theorem 1.4 is then proved.

Remark 2.23 An upper bound for $v(+\infty)$ can be derived from Proposition 2.4. Indeed, we have $v(0) \leq \frac{1-u(0)}{\min \{1, \Lambda\}}$, and since $v$ is nonincreasing, nontrivial, and $u(0)=\theta$, we get $v(+\infty)<v(0) \leq \frac{1-\theta}{\min \{1, \Lambda\}}$.

\subsection{Passage to the limit $\lambda \rightarrow 0$}

In this subsection, we study the behaviour of the two solutions $\left(u_{1}, v_{1}, c_{1}\right)$ and $\left(u_{2}, v_{2}, c_{2}\right)$ found above. Let us recall that, besides satisfying $(1-2),\left(u_{i}, v_{i}, c_{i}\right)$ satisfies

$$
u_{i}^{\prime}(0)=c_{i} u_{i}(0)+k\left(\theta, c_{i}, \lambda\right), u_{i}(0)=\theta, \Lambda v_{i}^{\prime}(0)=c_{i} v_{i}(0)-c_{i}
$$

with

$$
0 \leq c_{1} \leq r_{\lambda} \text { and } \frac{3 c_{*}}{4} \leq c_{2}
$$

and

$$
\|u\|_{C^{1}\left(I_{a}\right)} \leq 1+\sqrt{\frac{M}{\theta}}\left(1+\frac{1}{\underline{\Lambda}}\right),\|v\|_{C^{1}\left(I_{a}\right)} \leq 1+\frac{\sqrt{M}}{\sqrt{\theta} \Lambda},
$$

for $i=1,2$.

Let $\lambda \rightarrow 0$. Since $\left(u_{i}, v_{i}, c_{i}\right)$ is bounded independently of $\lambda$ in $C^{2}(\mathbb{R}) \times$ $C^{2}(\mathbb{R}) \times \mathbb{R}_{+}$from $(1)$ and $(50)$, by compactness it follows that $\left(u_{i}, v_{i}, c_{i}\right)$ converges, up to the extraction of some subsequence, in $C_{l o c}^{1}(\mathbb{R}) \times C_{l o c}^{1}(\mathbb{R}) \times \mathbb{R}_{+}$to a solution $\left(u_{i}^{0}, v_{i}^{0}, c_{i}^{0}\right)$ of

$$
\left\{\begin{aligned}
-\left(u_{i}^{0}\right)^{\prime \prime}+c_{i}^{0}\left(u_{i}^{0}\right)^{\prime} & =f\left(u_{i}^{0}, v_{i}^{0}\right) \\
-\Lambda\left(v_{i}^{0}\right)^{\prime \prime}+c_{i}^{0}\left(v_{i}^{0}\right)^{\prime} & =-f\left(u_{i}^{0}, v_{i}^{0}\right)
\end{aligned} \text { on } \mathbb{R},\right.
$$

with

$$
\left(u_{i}^{0}\right)^{\prime}(0)=c_{i}^{0} \theta, \Lambda\left(v_{i}^{0}\right)^{\prime}(0)=c_{i}^{0} v_{i}^{0}(0)-c_{i}^{0}, u_{i}^{0}(0)=\theta,
$$

since $k(\theta, c, 0)=0($ see $(18))$, and

$$
c_{1}^{0}=0, \frac{3 c_{*}}{4} \leq c_{2}^{0},
$$


Version définitive du manuscrit publié dans / Final version of the manuscript published in :

European Journal of Applied Mathematics, 2005, 16 (6), 741-765

since $\lim _{\lambda \rightarrow 0} r_{\lambda}=0$. Therefore, since $0<\frac{3 c_{*}}{4} \leq c_{2}^{0}$, Lemma 2.15 applied to $\left(u_{2}^{0}, v_{2}^{0}, c_{2}^{0}\right)$ gives

$$
u_{2}^{0}(+\infty)=1 \text { and } v_{2}^{0}(+\infty)=0 .
$$

Moreover, since for each $\lambda>0, u_{2} \leq \theta$ on $(-\infty, 0)$, we have

$$
\left\{\begin{aligned}
-\left(u_{2}^{0}\right)^{\prime \prime}+c_{2}^{0}\left(u_{2}^{0}\right)^{\prime} & =0 \\
-\Lambda\left(v_{2}^{0}\right)^{\prime \prime}+c_{2}^{0}\left(v_{2}^{0}\right)^{\prime} & =0
\end{aligned} \text { on }(-\infty, 0) .\right.
$$

Furthermore, $u_{2}$ is nondecreasing on $(-\infty, 0)$ and $v_{2}$ is nonincreasing on $(-\infty, 0)$; it follows that $u_{2}^{0}$ is nondecreasing on $(-\infty, 0)$ and $v_{2}^{0}$ is nonincreasing on $(-\infty, 0)$. Therefore, from (52) and (55),

$$
u_{2}^{0}(-\infty)=0 \text { and } v_{2}^{0}(-\infty)=1 .
$$

Hence, $\left(u_{2}^{0}, v_{2}^{0}, c_{2}^{0}\right)$ is a solution of $(8-9)$.

Let us study now $\left(u_{1}^{0}, v_{1}^{0}, c_{1}^{0}\right)$. As seen in (53), $c_{1}^{0}=0$. Since $u_{1} \geq 0$ and $v_{1} \geq 0$ for all $\lambda$, we have $u_{1}^{0} \geq 0$ and $v_{1}^{0} \geq 0$. It then follows from (51) that $u_{1}^{0}$ is concave. Since $u_{1}^{0}$ is bounded, it follows that $u_{1}^{0} \equiv u_{1}^{0}(0)=\theta$. Similarly, $v_{1}^{0} \equiv K_{1}$, where $K_{1}$ is an unknown constant.

Let us assume now that $\Lambda \leq 1$ and $g(y)=y$ on $\mathbb{R}$. Then, as mentioned in Remark 1.2 the problem (8-9) has a unique solution. Since we have just demonstrated that for all converging subsequences $\left(u_{2}, v_{2}, c_{2}\right)_{k}$ of $\left(u_{2}, v_{2}, c_{2}\right)$, $\left(u_{2}, v_{2}, c_{2}\right)_{k}$ converges to a solution of (8-9); this uniqueness result allows us to say that the whole sequence $\left(u_{2}, v_{2}, c_{2}\right)$ converges to the solution of (8-9).

Similarly, it follows from Remark 1.3 that, when $\Lambda=1$, for the reaction term $f(x, y)=p(x) g(y)$ satisfying (4-6), the whole sequence $\left(u_{2}, v_{2}, c_{2}\right)$ converges to the solution of (8-9).

That concludes the proof of Theorem 1.4, part 2).

\section{Proof of Theorem 1.5}

In this section, we give the proof of Theorem 1.5, establishing some results about the general solutions of (1-2).

\subsection{An upper bound for $\lambda$}

Let us prove that problem (1-2) has no solution for $\lambda$ large enough.

Lemma 3.1 Let $(u, v, c)$ be a nontrivial solution of (1-2) with $u \geq 0$ and $v \geq 0$. Then $c>0$.

Proof of Lemma 3.1: Assume by contradiction that $c=0$. Then, from $(1)$,

$$
\Lambda v^{\prime \prime}=f(u, v) \geq 0 \text { on } \mathbb{R},
$$

thus $v$ is a convex function. From (2), it follows that the function $v$ is constant. Hence, (57) gives $f(u, v)=0$, thus, from $(1),-u^{\prime \prime}=-\lambda h(u) \leq 0$. Similarly, 
Version définitive du manuscrit publié dans / Final version of the manuscript published in :

European Journal of Applied Mathematics, 2005, 16 (6), 741-765

it follows from the boundary conditions (2) that $u$ is a constant function. The solution $(u, v, c)$ is then trivial.

Furthermore, using similar arguments as in Lemmas 2.6 and 2.7, we obtain the following lemma.

Lemma 3.2 Let $(u, v, c)$ be a solution of (1-2) with $u \geq 0$ and $v \geq 0$. Then $u \leq 1, v \leq 1$ and $v$ is nonincreasing. Moreover, if $\Lambda \geq 1$ then $u \leq 1-v$.

Now, let us integrate the equation satisfied by $u$ between $-\infty$ and $+\infty$; we obtain, from (2),

$$
\int_{-\infty}^{+\infty} f(u, v)-\lambda \int_{-\infty}^{+\infty} h(u)=0 .
$$

(Indeed, $u^{\prime}( \pm \infty)=0$, see for instance Lemma 2.22). Moreover, from Lemma 3.2 , we have $0 \leq v \leq 1$ and $0 \leq u \leq 1$, therefore, using the hypotheses (3-7), we obtain that $f(u, v) \leq f(1,1) \frac{h(u)}{h(\theta)}$. Therefore, from (58), we deduce that

$$
\int_{-\infty}^{+\infty} f(1,1) \frac{h(u)}{h(\theta)} \geq \lambda \int_{-\infty}^{+\infty} h(u)
$$

and thus $\lambda \leq \frac{f(1,1)}{h(\theta)}$. This completes the proof of Theorem 1.5, part 1).

\subsection{A lower bound for the unburned gases}

In order to establish a lower bound we need some more computations. Assume that the function $g$ is Lipschitz-continuous on $\mathbb{R}$, and let $(u, v, c)$ be a nontrivial solution of (1-2).

Let us show first that $v(+\infty) \neq 0$. Since the solution $(u, v, c)$ is nontrivial, we can assume that it satisfies $u(0)=\theta$ (see Proposition 2.1). Thus we can define $x_{0}$, as the smallest $y \in[0,+\infty)$ such that $u(y)=\theta$ and $u \leq \theta$ for all $x \geq y$. Hence $(u, v, c)$ satisfies the following problem:

$$
\left\{\begin{array}{r}
-u^{\prime \prime}+c u^{\prime}+\lambda h(u)=0 \\
-\Lambda v^{\prime \prime}+c v^{\prime}=0
\end{array} \text { on }\left(x_{0},+\infty\right)\right.
$$

with the boundary conditions

$$
\left\{\begin{array}{l}
u\left(x_{0}\right)=\theta, \quad u(+\infty)=0 \\
v^{\prime}(+\infty)=0 .
\end{array}\right.
$$

It immediately follows that $v \equiv v(+\infty)$ on $\left(x_{0},+\infty\right)$. Moreover, using Proposition 2.1 (13), we know that $u^{\prime}\left(x_{0}\right)=c \theta-\lambda \int_{x_{0}}^{+\infty} h\left[u_{+}(s)\right] d s$, where $u_{+}$is the unique solution of

$$
\left\{\begin{array}{l}
-u_{+}^{\prime \prime}+c u_{+}^{\prime}+\lambda h\left(u_{+}\right)=0 \quad \text { on }\left(x_{0},+\infty\right) \\
u_{+}\left(x_{0}\right)=\theta, u_{+}(+\infty)=0 .
\end{array}\right.
$$




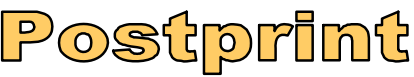

Version définitive du manuscrit publié dans / Final version of the manuscript published in :

European Journal of Applied Mathematics, 2005, 16 (6), 741-765

Also, if $v(+\infty)=0$, we see that

$$
v\left(x_{0}\right)=0, v^{\prime}\left(x_{0}\right)=0, u\left(x_{0}\right)=\theta \text { and } u^{\prime}\left(x_{0}\right)=c \theta-\lambda \int_{x_{0}}^{+\infty} h\left[u_{+}(s)\right] d s .
$$

It follows from the Cauchy-Lipschitz uniqueness theorem that $v \equiv 0$, and hence this is a contradiction. Therefore, we have shown that $v_{\infty}:=v(+\infty)>0$.

Now, dividing the second equation of (1) by $v$, and integrating by parts over $\mathbb{R}$, we obtain, using $v_{\infty}>0, v^{\prime} \leq 0$ (see Lemma 3.2) and (2),

$$
-\Lambda \int_{\mathbb{R}}\left[\frac{v^{\prime}}{v}\right]^{2}+c \ln \left(v_{\infty}\right)=-\int_{\mathbb{R}} \frac{f(u, v)}{v},
$$

and it follows that

$$
c \ln \left(v_{\infty}\right) \geq-\int_{\mathbb{R}} \frac{f(u, v)}{v} .
$$

Let $K$ be the Lipschitz constant of $g$. Then $\frac{g(v)}{v} \leq K$ on $\mathbb{R}$, thus, since $f(u, v)=p(u) g(v)$, we get

$$
c \ln \left(v_{\infty}\right) \geq-K \int_{\mathbb{R}} p(u)
$$

Since $p(u) \leq h(u) \frac{p(1)}{h(\theta)}$, it follows from (64) that

$$
c \ln \left(v_{\infty}\right) \geq-K \frac{p(1)}{h(\theta)} \int_{\mathbb{R}} h(u),
$$

and adding the equations in (1) and integrating over $\mathbb{R}$ we obtain

$$
\int_{\mathbb{R}} h(u)=\frac{c}{\lambda}\left(1-v_{\infty}\right) .
$$

From (65) and (66), we deduce that

$$
\ln \left(v_{\infty}\right) \geq-K \frac{p(1)}{\lambda h(\theta)}\left(1-v_{\infty}\right)>-\frac{K p(1)}{\lambda h(\theta)} .
$$

Finally one deduces that

$$
v_{\infty}>\exp \left(-\frac{K p(1)}{\lambda h(\theta)}\right)
$$

and part 2) of Theorem 1.5 is proved.

\subsection{Upper bounds for the flame speed $c$}

Let $x_{0}$ be defined as in the subsection 3.2. We can again assume (up to translation) that every solution $(u, v, c)$ of (1-2) satisfies $u(0)=\theta$ and $u \leq \theta$ on $(-\infty, 0)$. 
Version définitive du manuscrit publié dans / Final version of the manuscript published in :

European Journal of Applied Mathematics, 2005, 16 (6), 741-765

\subsubsection{Comparison with an adiabatic problem}

In this subsection, we assume that $\Lambda \geq 1$. As was proved in [3], we know that the following problem admits a unique solution $\left(u_{s}, c_{s}\right)$ :

$$
-\Lambda u_{s}^{\prime \prime}+c_{s} u_{s}^{\prime}=f\left(u_{s}, 1-u_{s}\right),
$$

with the boundary conditions

$$
u_{s}(-\infty)=0, u_{s}(+\infty)=1 \text { and } u_{s}(0)=\theta .
$$

Furthermore, $u_{s}$ is strictly increasing, therefore, setting $w=1-u_{s}$, we can define a function $k$ by $k(y):=-w^{\prime} \circ w^{-1}(1-y)$, moreover, $k \in C^{1}(0,1), k>0$ on $(0,1)$ and $k(\theta)=u_{s}^{\prime}(0)=\frac{c_{s} \theta}{\Lambda}$.

Then we need:

Lemma 3.3 Let $(u, v, c)$ be a solution of (1-2) with $c>0, u \geq 0$ and $v \geq 0$. Then $v$ is decreasing on $\left(-\infty, x_{0}\right)$.

Proof of Lemma 3.3: This is similar to that of Lemma 3.2, using $v^{\prime}\left(x_{0}\right)=0$.

Let us set $j(y):=-v^{\prime} \circ v^{-1}(1-y)$. Then, from Lemma $3.3, j$ is well defined and $j \in C^{1}\left(\left[1-v(0), 1-v_{\infty}\right]\right)$. Moreover, $j>0$ on $\left(1-v(0), 1-v_{\infty}\right)$, and

$$
j(1-v(0))=\frac{c}{\Lambda}(1-v(0)), j\left(1-v_{\infty}\right)=-v^{\prime}\left(x_{0}\right)=0 .
$$

The equation satisfied by $v$ in (1) gives

$$
-\Lambda v^{\prime \prime}\left(v^{-1}(1-y)\right)+c v^{\prime}\left(v^{-1}(1-y)\right)=-f\left(u\left(v^{-1}(1-y)\right), 1-y\right),
$$

for $y$ in $\left(1-v(0), 1-v_{\infty}\right)$. But, since $\Lambda \geq 1$, Lemma 3.2 gives $u \leq 1-v$, thus

$$
-\Lambda v^{\prime \prime}\left(v^{-1}(1-y)\right)+c v^{\prime}\left(v^{-1}(1-y)\right) \geq-f\left(1-v \circ v^{-1}(1-y), 1-y\right),
$$

for $y$ in $\left(1-v(0), 1-v_{\infty}\right)$, which finally gives

$$
\left(\Lambda j j^{\prime}-c j\right)(y) \geq-f(y, 1-y) \text { in }\left(1-v(0), 1-v_{\infty}\right) .
$$

Similarly, we have

$$
\left(\Lambda k k^{\prime}-c_{s} k\right)(y)=-f(y, 1-y) \text { in }(0,1) .
$$

Moreover, since $k>0$, we deduce from (72) that $\left(k(y)-\frac{c_{s} y}{\Lambda}\right)^{\prime}<0$, therefore integrating between $\theta$ and $1-v(0)$, we get

$$
k(1-v(0))<\frac{c_{s}}{\Lambda}(1-v(0))=\frac{c_{s}}{c} j(1-v(0)),
$$

by (70), thus

$$
\frac{k(1-v(0))}{j(1-v(0))}<\frac{c_{s}}{c}
$$




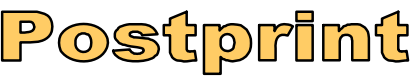

Version définitive du manuscrit publié dans / Final version of the manuscript published in :

European Journal of Applied Mathematics, 2005, 16 (6), 741-765

Then, subtracting equation (72) from (71), we obtain

$$
\frac{\Lambda}{2}\left(j^{2}-k^{2}\right)^{\prime} \geq c\left(j-\frac{c_{s}}{c} k\right) \text { in }\left(1-v(0), 1-v_{\infty}\right) .
$$

Now, assume that $\frac{c_{s}}{c}<1$, then, from (73) and (74),

$$
\begin{gathered}
\left(j^{2}-k^{2}\right)^{\prime}(1-v(0))>0, \text { and } \\
\left(j^{2}-k^{2}\right)(1-v(0))>0
\end{gathered}
$$

Let us assume now that the set $\left\{y \in\left(1-v(0), 1-v_{\infty}\right)\right.$ s.t. $\left.\left(j^{2}-k^{2}\right)(y)=0\right\}$ is nonempty and admits a lower bound $y_{1}$. Then $\left(j^{2}-k^{2}\right)\left(y_{1}\right)=0$ and, from (74), it follows that $\left(j^{2}-k^{2}\right)^{\prime}\left(y_{1}\right)>0$ which is impossible from the definition of $y_{1}$. Therefore, $j^{2}>k^{2}$ on $\left(1-v(0), 1-v_{\infty}\right)$, which is impossible since (70) gives $j\left(1-v_{\infty}\right)=0$, and $k>0$ in $(0,1)$. Finally, we obtain

$$
\frac{c_{s}}{c} \geq 1
$$

and Theorem 1.5, part 3), is proved.

\subsubsection{Computation of explicit upper bounds for $c$}

Let us assume that $\Lambda \geq 1$, and set

$$
\sigma_{1}=\max _{s \in(\theta, 1)} \frac{f(s, 1-s)}{s},
$$

we get, using (72),

$$
k^{\prime} \geq \frac{c_{s}}{\Lambda}-\frac{\sigma_{1} y}{\Lambda k(y)} \text { for } y \in(0,1) .
$$

Let us assume that $c_{s}>2 \sqrt{\sigma_{1} \Lambda}$. Now, as was done in [12] for bounded intervals, we can set $m(y)=r y$, with $r=\frac{c_{s}+\sqrt{c_{s}^{2}-4 \sigma_{1} \Lambda}}{2 \Lambda}$. Then we have the following:

Lemma $3.4 k(y)>m(y)$ for all $y \in(\theta, 1)$.

Proof of Lemma 3.4: First, let us note that

$$
m^{\prime}(y)=\frac{c_{s}}{\Lambda}-\frac{\sigma_{1} y}{\Lambda m(y)} \text { for } y \in(0,1)
$$

Therefore, since $m(\theta)=r \theta<\frac{c_{s}}{\Lambda} \theta$, and $k(\theta)=-w^{\prime}(0)=\frac{c_{s}}{\Lambda} \theta$, we see that $k(\theta)>m(\theta)$. Furthermore, from (79) and (80),

$$
(k-m)^{\prime}(y) \geq \frac{\sigma_{1} y}{\Lambda}\left(\frac{1}{m(y)}-\frac{1}{k(y)}\right) \text { for } y \in(\theta, 1) .
$$


Version définitive du manuscrit publié dans / Final version of the manuscript published in :

European Journal of Applied Mathematics, 2005, 16 (6), 741-765

It follows that $k(y)>m(y)$ on $(\theta, 1)$.

Hence, for all $x \in(0,+\infty), m(1-w(x))<k(1-w(x))$, thus $r(1-w(x))<$ $-w^{\prime}(x)$ which is equivalent to

$$
r<\frac{\left(u_{s}\right)^{\prime}(x)}{u_{s}(x)} \text { for } x \in(0,+\infty),
$$

where $u_{s}$ is defined by (68-69). Integrating (81) between 0 and $a>0$, we obtain

$$
\ln \left(\frac{u_{s}(a)}{\theta}\right)>r a .
$$

It follows from the definition of $r$ that, for $a$ chosen large enough, $c_{s}$ can be as small as we want, which is in contradiction to the hypothesis $c_{s} \geq 2 \sqrt{\sigma_{1} \Lambda}$. Finally, it follows that $c_{s}<2 \sqrt{\sigma_{1} \Lambda}$, and from (77) we have

$$
c<2 \sqrt{\sigma_{1} \Lambda}
$$

Let us now compute another upper bound for $c$, depending on $\theta$ but holding for all $\Lambda \geq 0$.

Let us set $\sigma_{2}=\max _{s \in[0,1]} f(1-\underline{\Lambda} s, s)$, and let $w$ be the solution of

$$
\left\{\begin{array}{l}
-w^{\prime \prime}+c w^{\prime}=\sigma_{2} \text { on }(0, a) \\
w^{\prime}(0)=c w(0)+k(\theta, c, \lambda) \\
w(a)=1
\end{array}\right.
$$

where $k(\theta, c, \lambda)$ is defined as in (14). The function $w$ can be computed explicitly:

$$
w(x)=X_{1}(a)+X_{2}(a) e^{c x}+\frac{\sigma_{2}}{c} x,
$$

where $X_{1}, X_{2}$ are two real-valued functions.

Moreover, $w^{\prime}(0)=c w(0)+k(\theta, c, \lambda)$ gives $X_{1}(a)=\frac{\sigma_{2}}{c^{2}}-\frac{k}{c}$, and $\lim _{a \rightarrow+\infty} X_{2}(a)=0$ since $w(a)=1$. Finally, we get $w(0)=X_{1}(a)+X_{2}(a) \rightarrow \frac{\sigma_{2}}{c^{2}}-\frac{k}{c}$ as $a \rightarrow+\infty$.

Since the function $w$ is a super-solution of the equation satisfied by $u$, we get, from the boundary conditions of $w$ together with the maximum principle, that $w \geq u$ on $(0, a)$, thus $w(0)=X_{1}(a)+X_{2}(a) \geq u(0)=\theta$. Therefore, taking $a$ large enough, we deduce that $c<\sqrt{\frac{\sigma_{2}}{\theta}}$.

The proof of Theorem 1.5 is complete.

\section{Numerical results}

In this section we give some numerical approximations of the two solutions obtained in Corollary 2.21 on a bounded domain $[0, a]$. Numerically, no other 


\section{Version définitive du manuscrit publié dans / Final version of the manuscript} published in :

European Journal of Applied Mathematics, 2005, 16 (6), 741-765
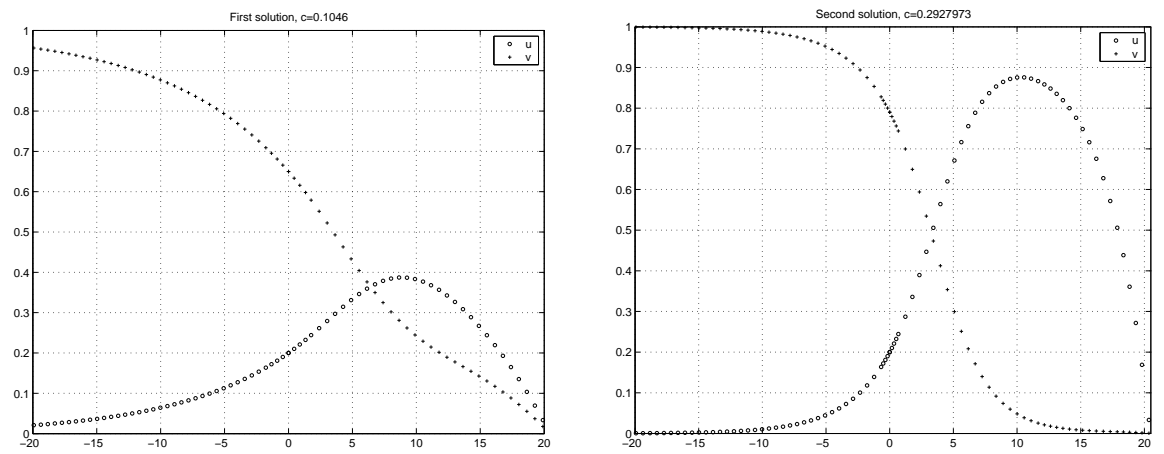

Figure 1: First solution: $c=0.10$, and second solution: $c=0.29$

solution has been found. A shooting method on $c$ and $v(0)$ was used to compute these approximations, and the solutions were extended to $[-a, a]$.

We have taken here $f(u, v)=v H(u-\theta)(u-\theta)^{2}$, where $H$ is the Heaviside function, and $h(u)=u$. The values of the parameters are: $\Lambda=1, \lambda=0.01$, $\theta=0.2$ and $a=20$. The results are plotted in Figure 1.

\section{Concluding remarks}

Besides providing an existence result, Theorem 1.4 also solves a non-uniqueness problem. In the adiabatic case Marion [12] has proved the uniqueness of flames when the Lewis number $(1 / \Lambda$ in this paper) is greater than 1 , and Bonnet [4] has shown that when the Lewis number is less than unity, uniqueness cannot be generally assumed. Here, we prove that uniqueness never holds in the nonadiabatic case (for small heat losses $\lambda$ ). When heat loss intensity goes to zero one of our solutions has a flame velocity $c$ which goes to zero. The asymptotic behaviour of the other solution, as $\lambda \rightarrow 0$ could depend on the value of the Lewis number. Indeed, the result of Theorem 1.4 part 2), which asserts that this solution goes to the adiabatic solution as $\lambda \rightarrow 0$, may not be true in the nonuniqueness case of Bonnet [4]. Nevertheless, the counter-example to uniqueness of [4] involves a special type of nonlinearity, and even if the Lewis number is less than unity, there are many examples of nonlinearities for which uniqueness holds in the adiabatic framework.

Theorem 1.5 provides some estimates for every solution $(u, v, c)$ of (1-2), for all $\lambda>0$. These results include the case of non-unit Lewis numbers and can therefore be used in many physically meaningful situations.

Even if the present paper gives some answers about the non-adiabatic combustion model, some questions remain open. We do not know if uniqueness holds for each fixed value of $c$; the curve $c \mapsto \lambda(c)$ would be then bell-shaped $(\lambda(c)$ is bounded from part 1) of Theorem 1.5). Similarly, the question of the existence of a critical value $\lambda^{*}$ such that a solution $(u, v, c)$ exists if and only if $\lambda \leq \lambda^{*}$ 
Version définitive du manuscrit publié dans / Final version of the manuscript published in :

European Journal of Applied Mathematics, 2005, 16 (6), 741-765

is still open. In the adiabatic case, the stability of the solution, when $\Lambda=1$ has been treated by Berestycki, Larrouturou \& Roquejoffre [2] (linear stability) and Roquejoffre [15] (nonlinear stability). Here, the analysis of the stability is even more complicated, since the system (1-2) cannot reduce to a scalar equation, even if $\Lambda=1$. However, we conjecture, from our numerical computations and from the asymptotic analysis of $[9,10]$, that the solution with small flame velocity is unstable, whereas the other is stable.

\section{Acknowledgements}

I am very grateful to Professor Henri Berestycki who suggested I develop this research work. I would like to thank him, and Professor François Hamel as well, for their interest in my work and for the fruitful discussions we had on this problem. It is also a pleasure to thank the editor and the anonymous referees for their valuable comments.

\section{Appendix}

Let us recall the notation of Proposition 2.16:

$$
\begin{aligned}
\phi(c) & =\frac{c}{\lambda-1}\left(\frac{1}{e^{a \Delta_{\lambda}} r_{2}+r_{2}-c}+\frac{1}{e^{-a \Delta_{\lambda}} r_{1}+r_{1}-c}\right. \\
& \left.-\frac{1}{e^{a \Delta} z_{2}+z_{2}-c}-\frac{1}{e^{-a \Delta} z_{1}+z_{1}-c}\right),
\end{aligned}
$$

and $\Delta_{\lambda}:=\sqrt{c^{2}+4 \lambda}, \Delta:=\sqrt{c^{2}+4}, r_{1}=\frac{c-\Delta_{\lambda}}{2}, r_{2}=\frac{c+\Delta_{\lambda}}{2}, z_{1}=\frac{c-\Delta}{2}$, and $z_{2}=\frac{c+\Delta}{2}$. It is straightforward to show that $\phi$ is a $C^{2}([0,+\infty))$ function. Set $\phi^{\prime}:=\frac{\partial}{\partial c} \phi$.

Proposition 5.1 For $\lambda<1$ and a large enough, $\phi^{\prime}$ vanishes only once on $(0,+\infty)$

Proof of Proposition 5.1: Let us show at first that

Lemma 5.2 Let $\mu$ be the function such that, for a large enough and for all $c$ in $(0,+\infty), \phi(c)=\frac{2}{1-\lambda}\left(\frac{c}{c+\Delta_{\lambda}}-\frac{c}{c+\Delta}\right)+\mu(c, a)$. Then the function $\mu_{a}(c):=c \mapsto$ $\mu(c, a)$ is in $C^{2}([0,+\infty))$ for a large enough and

$$
\left|(\mu(c, a))^{(n)}\right| \leq \frac{4}{1-\lambda} e^{\frac{-a \Delta_{\lambda}}{2}} \text { for all } n \in\{0,1,2\}
$$

where $(\mu(c, a))^{(n)}$ is the $n^{\text {th }}$ derivative of the function $\mu$ with respect to the variable $c$. 
Version définitive du manuscrit publié dans / Final version of the manuscript published in :

European Journal of Applied Mathematics, 2005, 16 (6), 741-765

Proof of Lemma 5.2: Setting $\mu(c, a):=\phi(c)-\frac{2}{1-\lambda}\left(\frac{c}{c+\Delta_{\lambda}}-\frac{c}{c+\Delta}\right)$, it easily follows that $c \mapsto \mu(c, a)$ is a $C^{2}([0,+\infty))$ function for $a$ large enough. Furthermore, we see that

$$
\left|\left(\frac{c}{e^{a \Delta_{\lambda}} r_{2}+r_{2}-c}\right)^{(n)}\right| \leq e^{-\frac{a \Delta_{\lambda}}{2}} \text { and }\left|\left(\frac{c}{e^{a \Delta_{2}} z_{2}+z_{2}-c}\right)^{(n)}\right| \leq e^{-\frac{a \Delta_{\lambda}}{2}},
$$

for all $n \in\{0,1,2\}$. Furthermore, we can also easily check that

$$
\left|\left(\frac{2 c}{c+\Delta_{\lambda}}+\frac{c}{e^{-a \Delta_{\lambda}} r_{1}+r_{1}-c}\right)^{(n)}\right| \leq e^{-\frac{a \Delta_{\lambda}}{2}} \text {, for all } n \in\{0,1,2\},
$$

and the same inequality holds with $\Delta_{\lambda}$ replaced by $\Delta$ and $r_{1}$ replaced by $z_{1}$.

Finally, Lemma 5.2 follows from the above calculations.

In the following, we write $\mu^{\prime}(c, a)$ for $\frac{\partial \mu}{\partial c}(c, a)$, and $\mu^{\prime \prime}(c, a)$ for $\frac{\partial^{2} \mu}{\partial c^{2}}(c, a)$.

Let us set $b(c):=\frac{c}{c+\Delta_{\lambda}}-\frac{c}{c+\Delta}$.

Lemma 5.3 The first derivative of $b$ vanishes only once on $(0,+\infty)$.

Proof of Lemma 5.3: Let us notice at first that $\Delta_{\lambda}^{\prime}=\frac{c}{\Delta_{\lambda}}, \Delta^{\prime}=\frac{c}{\Delta}, \Delta_{\lambda}^{\prime \prime}=\frac{1}{\Delta_{\lambda}}-\frac{c^{2}}{\Delta_{\lambda}^{3}}$ and $\Delta^{\prime \prime}=\frac{1}{\Delta}-\frac{c^{2}}{\Delta^{3}}$. Then we get

$$
b^{\prime}(c)=\frac{c+\Delta_{\lambda}-c\left(1+\Delta_{\lambda}^{\prime}\right)}{\left(c+\Delta_{\lambda}\right)^{2}}-\frac{c+\Delta-c\left(1+\Delta^{\prime}\right)}{(c+\Delta)^{2}}=\frac{\Delta_{\lambda}-\frac{c^{2}}{\Delta_{\lambda}}}{\left(c+\Delta_{\lambda}\right)^{2}}-\frac{\Delta-\frac{c^{2}}{\Delta}}{(c+\Delta)^{2}} .
$$

Setting $k(c)=b^{\prime}(c)\left(c+\Delta_{\lambda}\right)^{2}(c+\Delta)^{2}$, we obtain

$$
k(c)=\left(\Delta_{\lambda}-\frac{c^{2}}{\Delta_{\lambda}}\right)(c+\Delta)^{2}-\left(\Delta-\frac{c^{2}}{\Delta}\right)\left(c+\Delta_{\lambda}\right)^{2},
$$

thus we have $k^{\prime}(c)=2(c+\Delta)\left(1+\frac{c}{\Delta}\right)\left(\Delta_{\lambda}-\frac{c^{2}}{\Delta_{\lambda}}\right)-2\left(c+\Delta_{\lambda}\right)\left(1+\frac{c}{\Delta_{\lambda}}\right)\left(\Delta-\frac{c^{2}}{\Delta}\right)$ $+c\left[\left(-\frac{1}{\Delta_{\lambda}}+\frac{c^{2}}{\Delta_{\lambda}^{3}}\right)(c+\Delta)^{2}+\left(\frac{1}{\Delta}-\frac{c^{2}}{\Delta^{3}}\right)\left(c+\Delta_{\lambda}\right)^{2}\right]$. Hence, $k^{\prime}(c)=5 c^{3}\left(\frac{1}{\Delta}-\frac{1}{\Delta_{\lambda}}\right)+4 c\left(\Delta_{\lambda}-\Delta\right)+6 c^{2}\left(\frac{\Delta_{\lambda}}{\Delta}-\frac{\Delta}{\Delta_{\lambda}}\right)+c^{5}\left(\frac{1}{\Delta^{3}}-\frac{1}{\Delta_{\lambda}^{3}}\right)+2 c^{4}\left(\frac{\Delta}{\Delta_{\lambda}^{3}}-\frac{\Delta_{\lambda}}{\Delta^{3}}\right)+$ $c^{3}\left(\frac{\Delta^{2}}{\Delta_{\lambda}^{3}}-\frac{\Delta_{\lambda}^{2}}{\Delta^{3}}\right)+c\left(\frac{\Delta_{\lambda}^{2}}{\Delta}-\frac{\Delta^{2}}{\Delta_{\lambda}}\right)$. Therefore, we can write $k^{\prime}(c)=c\left(\frac{1}{\Delta_{\lambda}}-\frac{1}{\Delta}\right) I(c)$, with

$$
\begin{aligned}
I(c) & =-5 c^{2}-4 \Delta \Delta_{\lambda}-6 c\left(\Delta_{\lambda}+\Delta\right)+c^{4}\left(\frac{1}{\Delta_{\lambda}^{2}}+\frac{1}{\Delta \Delta_{\lambda}}+\frac{1}{\Delta^{2}}\right) \\
& +2 c^{3}\left(\frac{\Delta}{\Delta_{\lambda}^{2}}+\frac{1}{\Delta_{\lambda}}+\frac{1}{\Delta}+\frac{\Delta_{\lambda}}{\Delta^{2}}\right)+c^{2}\left(\frac{\Delta^{2}}{\Delta_{\lambda}^{2}}+\frac{\Delta}{\Delta_{\lambda}}+1+\frac{\Delta_{\lambda}}{\Delta}+\frac{\Delta_{\lambda^{2}}}{\Delta^{2}}\right) \\
& -\left(\Delta_{\lambda}^{2}+\Delta \Delta_{\lambda}+\Delta^{2}\right) .
\end{aligned}
$$


Version définitive du manuscrit publié dans / Final version of the manuscript published in :

European Journal of Applied Mathematics, 2005, 16 (6), 741-765

Furthermore, since $c<\Delta_{\lambda}<\Delta$, we see that $I(c) \leq-8 c^{2}$. Finally,

$$
k^{\prime}(c) \leq-8 c^{3}\left(\frac{1}{\Delta_{\lambda}}-\frac{1}{\Delta}\right)<0 .
$$

Furthermore, from a series expansion of $k(c)$ at $c=+\infty$, we obtain

$$
\lim _{c \rightarrow+\infty} k(c)=8(\lambda-1)<0,
$$

and, since

$$
k(0)=8 \sqrt{\lambda}-8 \lambda=8 \sqrt{\lambda}(1-\sqrt{\lambda})>0,
$$

we deduce from (85-87) that $k$ vanishes only once, and since $k(c)=b^{\prime}(c)(c+$ $\left.\Delta_{\lambda}\right)^{2}(c+\Delta)^{2}$, Lemma 5.3 is proved.

Let us now prove Proposition 5.1.

We have $\phi^{\prime}(c)=\frac{2}{1-\lambda} b^{\prime}(c)+\mu^{\prime}(c, a)$, thus, from a straightforward computation,

$$
\phi^{\prime}(0)=\frac{1-\sqrt{\lambda}}{\sqrt{\lambda}(1-\lambda)}+\mu^{\prime}(0, a) .
$$

Therefore, we deduce from Lemma 5.2 that for $a$ large enough,

$$
\phi^{\prime}(0)>\frac{1}{2} \frac{1-\sqrt{\lambda}}{\sqrt{\lambda}(1-\lambda)}>0 .
$$

Moreover, it follows from (86) that

$$
b^{\prime}(c)-\frac{\lambda-1}{2 c^{4}} \rightarrow 0 \text { as } c \rightarrow+\infty .
$$

Thus, as a consequence of Lemma 5.2, we have $\phi^{\prime}(c)<-\frac{1}{2 c^{4}}<0$ for $c$ large enough. Hence, by continuity, $\phi^{\prime}$ vanishes at least once on $(0,+\infty)$.

From (87) and (89), we know that $b^{\prime}(0)>0$, and $b^{\prime}(c)<0$ for $c$ large enough. Therefore, it follows from Lemma 5.3 that there exists a unique $c_{0}>0$ such that $b^{\prime}>0$ on $\left[0, c_{0}\right) b^{\prime}\left(c_{0}\right)=0$ and $b^{\prime}<0$ on $\left(c_{0},+\infty\right)$. Let $c_{1}$ and $c_{2}$ be two points in $\mathbb{R}_{+}$such that $\phi^{\prime}\left(c_{1}\right)=\phi^{\prime}\left(c_{2}\right)=0$. Then

$$
\left|b^{\prime}\left(c_{1}\right)\right| \leq \frac{1-\lambda}{2}\left|\mu^{\prime}\left(c_{1}, a\right)\right| .
$$

Using Lemma 5.3 and the continuity of $b^{\prime},\left|c_{0}-c_{1}\right| \rightarrow 0$ as $b^{\prime}\left(c_{*}\right) \rightarrow 0$. Hence Lemma 5.2 and (90) give

$$
\left|c_{0}-c_{1}\right| \rightarrow 0 \text { as } a \rightarrow+\infty .
$$

Assume that $c_{1} \neq c_{2}$ and set $l(c):=\frac{1-\lambda}{2} \phi^{\prime}(c)\left(c+\Delta_{\lambda}\right)^{2}(c+\Delta)^{2}$, then $l\left(c_{1}\right)=$ $l\left(c_{2}\right)=0$ and there exists $c_{3} \in\left[c_{1}, c_{2}\right]$ such that $l^{\prime}\left(c_{3}\right)=0$, since $c_{1} \neq c_{2}$. Thus, since

$$
l(c)=k(c)+\frac{1-\lambda}{2}\left(c+\Delta_{\lambda}\right)^{2}(c+\Delta)^{2} \mu^{\prime}(c, a)
$$


Version définitive du manuscrit publié dans / Final version of the manuscript published in :

European Journal of Applied Mathematics, 2005, 16 (6), 741-765

we have $k^{\prime}\left(c_{3}\right)=t_{1}\left(c_{3}\right) \mu^{\prime}\left(c_{3}, a\right)+t_{2}\left(c_{3}\right) \mu^{\prime \prime}\left(c_{3}, a\right)$, where $t_{1}$ and $t_{2}$ are two functions which are polynomially increasing.

From (91), we can take $a$ large enough such that $\left|c_{0}-c_{1}\right|<\frac{c_{0}}{2}$, and, since $c_{0}>0, c_{1}>\frac{c_{0}}{2}$. Thus, since (85) gives $k^{\prime}(c) \leq-8 c^{3}\left(\frac{1}{\Delta_{\lambda}}-\frac{1}{\Delta}\right)$, we have

$$
k^{\prime}\left(c_{3}\right) \leq \max _{c>\frac{c_{0}}{2}}\left\{-8 c^{3}\left(\frac{1}{\Delta_{\lambda}(c)}-\frac{1}{\Delta(c)}\right)\right\} \leq-c_{0}^{3}\left(\frac{1}{\Delta_{\lambda}\left(\frac{c_{0}}{2}\right)}-\frac{1}{\Delta\left(\frac{c_{0}}{2}\right)}\right)<0 .
$$

But, from Lemma 5.2, we can assume for $a$ large enough that

$$
\left|k^{\prime}\left(c_{3}\right)\right|=\left|t_{1}\left(c_{3}\right) \mu^{\prime}\left(c_{3}, a\right)+t_{2}\left(c_{3}\right) \mu^{\prime \prime}\left(c_{3}, a\right)\right|<\frac{c_{0}^{3}}{2}\left(\frac{1}{\Delta_{\lambda}\left(\frac{c_{0}}{2}\right)}-\frac{1}{\Delta\left(\frac{c_{0}}{2}\right)}\right),
$$

therefore, (92) gives $c_{0}^{3}\left(\frac{1}{\Delta_{\lambda}\left(\frac{c_{0}}{2}\right)}-\frac{1}{\Delta\left(\frac{c_{0}}{2}\right)}\right)<\frac{c_{0}^{3}}{2}\left(\frac{1}{\Delta_{\lambda}\left(\frac{c_{0}}{2}\right)}-\frac{1}{\Delta\left(\frac{c_{0}}{2}\right)}\right)$. Thus, we get a contradiction, and we conclude that $c_{1}=c_{2}$. Proposition 5.1 is then proved.

Proposition 5.4 There exists $c_{\theta}>0$ such that for $\lambda$ small enough and a large enough, the equation $\phi(c)=\theta$ admits exactly two solutions $c^{1}$ and $c^{2}$, with $0<c^{1}<(2 \lambda)^{1 / 3}$ and $c^{2}>c_{\theta}$.

Proof of Proposition 5.4: Let us begin with the lemma:

Lemma 5.5 The function $\phi$ admits a unique maximum.

Proof of Lemma 5.5: We have seen in (88) that $\phi^{\prime}(0)>0$ for $a$ large enough. Moreover, $\phi(0)=0$ and $\phi(+\infty)=0$. The proof of Lemma 5.5 then follows from Proposition 5.1.

Let us now compute $\phi\left[(2 \lambda)^{1 / 3}\right]$. A series expansion about $\lambda=0$ gives

$$
\phi\left[(2 \lambda)^{1 / 3}\right]=1-\frac{3}{2^{2 / 3}} \lambda^{1 / 3}+\mu(\sqrt{\lambda}, a) .
$$

Therefore, for $a$ large enough and $\lambda$ small enough, $\phi\left[(2 \lambda)^{1 / 3}\right]$ can be as close as we want to 1 . It then follows from Lemma 5.5, with $\phi(0)=0, \phi(+\infty)=0$ and $\theta<1$, that the equation $\phi(c)=\theta$ admits exactly two solutions $c^{1}$ and $c^{2}$, with $0<c^{1}<(2 \lambda)^{1 / 3}$.

Next, a series expansion of $\phi(c)$ about $\lambda=0$ gives

$$
\phi(c)=\frac{\sqrt{c^{2}+4}-c}{\sqrt{c^{2}+4}+c}+O(\lambda)+\mu(\sqrt{\lambda}, a),
$$

and, with another series expansion at $c=0$, we obtain

$$
\phi(c)=1-c+O\left(c^{2}\right)+O(\lambda)+\mu(\sqrt{\lambda}, a),
$$

thus, since $\theta<1$, there exists $c_{\theta}>0$ independent of $\lambda$ and $a$, such that for $\lambda$ small enough and $a$ large enough, $\phi\left(c_{\theta}\right)>\theta$. It then follows from Lemma 5.5 that $c^{2}>c_{\theta}$. Proposition 5.4 follows. 
Version définitive du manuscrit publié dans / Final version of the manuscript published in :

European Journal of Applied Mathematics, 2005, 16 (6), 741-765

\section{References}

[1] Aftalion, A. \& Berestycki, H. (1998) Mathematical model of stagnation point flames. Preprint LMENS-98-25.

[2] Berestycki, H., Larrouturou, B. \& Roquejoffre J.-M. (1991) Stability of travelling fronts in a curved flame model. Part I: Linear analysis. Arch. Rat. Mech. Anal. 117, 97-117.

[3] Berestycki, H., Nicolaenko, B. \& Scheurer, B. (1985) Traveling wave solutions to combustion models and their singular limits. SIAM J. Math. Anal. 16, 1207-1242.

[4] Bonnet, A. (1995) Non-uniqueness for flame propagation when Lewis number is less than 1. Euro. Jnl of Applied Mathematics 6, 287-306.

[5] Gilbarg, D. \& Trudinger, NS. (1977) Elliptic Partial Differential Equations of Second Order, 224. Springer-Verlag.

[6] Giovangigli, V. (1990) Nonadiabatic plane laminar flames and their singular limits. SIAM J. Math. Anal. 21 (5), 1305-1325.

[7] Glangetas, L. \& Roquejoffre, J.M. (1995) Rigorous derivation of the dispersion relation in a combustion model with heat losses. Preprint Publications du Laboratoire d'Analyse Numérique R95032.

[8] Hosono, Y. \& Ilyas, B. (1995) Traveling waves for a simple diffusive epidemic model. Mathematical Models and Methods in Applied Sciences 5 (7), 935-966.

[9] Joulin, G. \& Clavin, P. (1976) Analyse asymptotique des conditions d'extinction des flammes laminaires. Acta Astronautica 3, 223-240.

[10] Joulin, G. \& Clavin, P. (1979) Linear stability of non adiabatic flames: diffusional thermal model. Combust. Flame 35137.

[11] Lions, P.L. (1982) On the existence of positive solutions of semilinear elliptic equations. SIAM J. Math. Anal. 24 (4), 441-467.

[12] Marion, M. (1985) Qualitative properties of a nonlinear system for laminar flames without ignition temperature. Nonlinear Analysis, Theory, Methods and Applications 9 (11), 1269-1292.

[13] Protter, M. H. \& Weinberger, H. F. (1967) Maximum Principles in Differential Equations, Prentice-Hall, New Jersey.

[14] Rabinowitz, P. H. (1974) Pairs of positive solutions of nonlinear elliptic boundary value problems. Indiana Univ. Math. J. 23, 729-754.

[15] Roquejoffre, J.-M. (1992) Stability of travelling fronts in a model for ame propagation. II. Nonlinear stability. Arch. Rat. Mech. Anal. 117, 119-153.

[16] Zel'dovich, Ya. B. (1941) A theory of the limit of slow flame propagation. Zh. Prikl. Mekh. $i$ Tekhn. Fiz. 11 (1), 159-169 (Russian). 